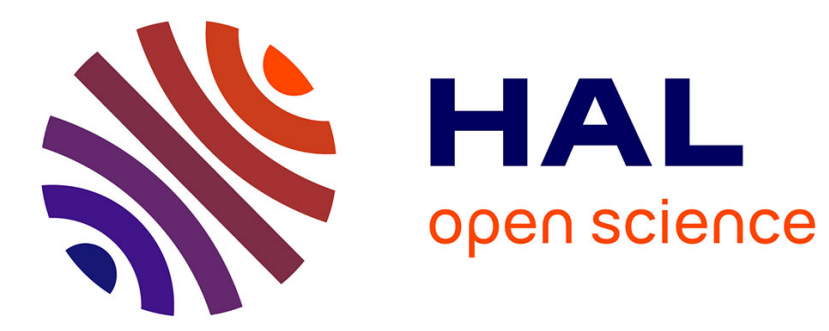

\title{
List colorings with measurable sets
}

Jan Hladky, Daniel Král', Jean-Sébastien Sereni, Michael Stiebitz

\section{To cite this version:}

Jan Hladky, Daniel Král', Jean-Sébastien Sereni, Michael Stiebitz. List colorings with measurable sets. Journal of Graph Theory, 2008, 59 (3), pp.229-238. 10.1002/jgt.20335 . hal-00487293

\section{HAL Id: hal-00487293 \\ https://hal.science/hal-00487293}

Submitted on 28 May 2010

HAL is a multi-disciplinary open access archive for the deposit and dissemination of scientific research documents, whether they are published or not. The documents may come from teaching and research institutions in France or abroad, or from public or private research centers.
L'archive ouverte pluridisciplinaire HAL, est destinée au dépôt et à la diffusion de documents scientifiques de niveau recherche, publiés ou non, émanant des établissements d'enseignement et de recherche français ou étrangers, des laboratoires publics ou privés. 


\title{
List colorings with measurable sets
}

\author{
Jan Hladký* Daniel Král’ ${ }^{\prime} \quad$ Jean-Sébastien Sereni ${ }^{\ddagger}$ \\ Michael Stiebitz ${ }^{\S}$
}

\begin{abstract}
The measurable list chromatic number of a graph $G$ is the smallest number $\xi$ such that if each vertex $v$ of $G$ is assigned a set $L(v)$ of measure $\xi$ in a fixed atomless measure space, then there exist sets $c(v) \subseteq L(v)$ such that each $c(v)$ has measure one and $c(v) \cap c\left(v^{\prime}\right)=\emptyset$ for every pair of adjacent vertices $v$ and $v^{\prime}$. We provide a simpler proof of a measurable generalization of Hall's theorem due to Hilton and Johnson [J. Graph Theory 54 (2007), 179-193] and show that the measurable list chromatic number of a finite graph $G$ is equal to its fractional chromatic number.
\end{abstract}

\section{Introduction}

In this paper, we study colorings and list colorings of graphs with measurable sets. This concept extends the standard notion of fractional colorings, which

*Department of Applied Mathematics (KAM), Faculty of Mathematics and Physics, Charles University, Malostranské náměstí 25, 11800 Prague, Czech Republic. E-mail: hladky@kam.mff.cuni.cz. This research was partially supported by grant KONTAKT ME 885.

$\dagger$ Institute for Theoretical Computer Science (ITI), Faculty of Mathematics and Physics, Charles University, Malostranské náměstí 25, 11800 Prague, Czech Republic. E-mail: kral@kam.mff.cuni.cz. The Institute for Theoretical Computer Science is supported by Ministry of Education of the Czech Republic as project 1M0545. This research was partially supported by grant KONTAKT ME 885.

${ }^{\ddagger}$ Institute for Theoretical Computer Science (ITI) and Department of Applied Mathematics (KAM), Faculty of Mathematics and Physics, Charles University, Malostranské náměstí 25, 11800 Prague, Czech Republic. E-mail: sereni@kam.mff.cuni.cz. This author is supported by the European project IST FET AEOLUs.

$\S$ Technische Universität Ilmenau, Institute of Mathematics, P.O.B. 100 565, D-98684 Ilmenau, Germany. E-mail: stieb@mathematik.tu-ilmenau.de. 
we recall now. A $q$-coloring of a graph $G$ with $p$ colors is a mapping that assigns to each vertex a subset of $\{1,2, \ldots, p\}$ of size $q$ such that the sets assigned to adjacent vertices are disjoint. The fractional chromatic number $\chi_{f}(G)$ of $G$ is the infimum of the values of $p / q$ taken over all $p$ and $q$ for which $G$ admits a $q$-coloring with $p$ colors. It is well-known $[8$, p. 24] that if $G$ is finite, then the infimum is always attained and thus $\chi_{f}(G)$ is rational. Fractional colorings form a prominent topic of graph theory, to which a whole book [8] is devoted.

We now consider an extension of fractional colorings introduced by Hilton and Johnson [5]. Let $(X, \mathcal{M}, \mu)$ be an atomless measure space, i.e., $\mathcal{M}$ is a $\sigma$-algebra on a set $X$, and $\mu$ a finite measure defined on $\mathcal{M}$ such that, for each $A \in \mathcal{M}$ with $\mu(A)>0$, there exists $B \subset A$ such that $B \in \mathcal{M}$ and $0<\mu(B)<\mu(A)$. A measurable coloring of a graph $G$ over the space $(X, \mathcal{M}, \mu)$ is a mapping $c: V(G) \rightarrow \mathcal{M}$ such that $\mu(c(v)) \geq 1$ for every $v \in V(G)$ and $c(v) \cap c\left(v^{\prime}\right)=\emptyset$ for every pair of adjacent vertices $v$ and $v^{\prime}$. The measurable chromatic number $\chi_{m}(G)$ of $G$ is the infimum of $\mu(X)$ over all atomless measurable spaces $(X, \mathcal{M}, \mu)$ over which $G$ admits a measurable coloring. This notion is indeed a "continuous" extension of fractional colorings as $\chi_{f}(G)=\chi_{m}(G)$ for finite graphs $G$. We note that a graph $G$ has a measurable coloring over $(X, \mathcal{M}, \mu)$ for every atomless measurable space $(X, \mathcal{M}, \mu)$ with $\mu(X) \geq \chi_{m}(G)$.

A list variant of fractional colorings was studied by Alon, Tuza and Voigt [1]. Fix a graph $G$. For each positive integer $q$, let $p_{q}$ be the smallest integer such that if every vertex $v$ is assigned a list $L(v)$ of $p_{q}$ colors, then there exists a $q$-coloring $c$ of $G$ with $c(v) \subseteq L(v)$. The list fractional chromatic number $\chi_{f, \ell}(G)$ of a graph $G$ is the limit of the ratio $p_{q} / q$ when $q$ tends to infinity. Alon et al. [1] showed that if $G$ is finite then $\chi_{f, \ell}(G)$ exists and is equal to $\chi_{f}(G)$. It seems unavoidable to consider the limit when $q$ tends to infinity in this definition. The reader can consult the book by Scheinerman and Ullman [8] for further details.

We believe that measurable colorings are a natural extension of fractional colorings, in particular when related to list colorings since this notion allows us to avoid the limit in the definition of the list fractional chromatic number. Let us define measurable list colorings formally. The measurable list chromatic number $\chi_{m, \ell}(G)$ of a graph $G$ is the infimum of all positive reals $\alpha$ such that if each vertex $v$ of $G$ is assigned a set $L(v) \in \mathcal{M}$ with $\mu(L(v))=\alpha$, then there exists a measurable coloring $c: V(G) \rightarrow \mathcal{M}$ such that $c(v) \subseteq L(v)$. A justification that this notion is a "continuous" list variant of fractional color- 
ings is the following statement, which we prove in Section 4: $\chi_{m, \ell}(G)=\chi_{f}(G)$ for every finite graph $G$. Hence, for every finite graph $G$, all the four quantities $\chi_{f}(G), \chi_{f, \ell}(G), \chi_{m}(G)$ and $\chi_{m, \ell}(G)$ are equal. As we note at the end of the paper, this is not true in general for infinite graphs.

We also give a new proof of a result of Hilton and Johnson [5], which generalizes an extension of Hall's theorem by Cropper et al. [3] as we outline in the next subsection. We believe that our proof is conceptually simpler since we restrict ourselves to using only elementary results from the measure theory and avoid using the Krein-Milman theorem (finding such a proof is mentioned as an open problem by Hoffman and Johnson [6, p. 11]).

\subsection{Measurable extension of Hall's theorem}

In this subsection, we introduce a theorem of Cropper, Gyarfás and Lehel [3], which is a generalization of Hall's theorem, and we discuss its extension to measurable colorings.

Cropper et al. [3] characterized the class of graphs that have the property that a Hall-type condition is sufficient for the existence of a multicoloring. Formally, if $\kappa: V(G) \rightarrow \mathbb{N}$ is a function that specifies the numbers of colors each vertex should be assigned and $L: V(G) \rightarrow 2^{X}$ is a list assignment, we say that the Hall condition is fulfilled if

$$
\sum_{v \in V(H)} \kappa(v) \leq \sum_{x \in X} \alpha(x, L, H) \text { for every subgraph } H \subseteq G,
$$

where $\alpha(x, L, H)$ is the independence number of the subgraph of $H$ induced by the vertices $v$ with $x \in L(v)$. A color $x$ can be assigned to at most $\alpha(x, L, H)$ vertices of $H$ and thus the condition (1) is necessary for the existence of a coloring. Observe that it is actually enough to verify the condition (1) for induced subgraphs $H$ of $G$. The following theorem provides a characterization of graphs for which the Hall condition is also sufficient for the existence of a coloring. We let $K_{4}^{-}$be the graph obtained from $K_{4}$ by removing an edge.

Theorem 1 (CGL Theorem [3]). The following statements are equivalent for a finite graph $G$.

- For every $\kappa: V(G) \rightarrow \mathbb{N}$ and $L: V(G) \rightarrow 2^{X}$, the Hall condition (1) is sufficient and necessary for the existence of a coloring $c$ such 
that for every $v \in V(G), c(v)$ is a subset of $L(v)$ of size $\kappa(v)$, and $c(v) \cap c\left(v^{\prime}\right)=\emptyset$ for every pair of adjacent vertices $v$ and $v^{\prime}$;

- $G$ is the line graph of a forest;

- every block of $G$ is a clique and each cut-vertex belongs exactly to two blocks;

- $G$ does not contain $K_{4}^{-}, K_{1,3}$ or a cycle of length more than 3 as an induced subgraph.

Note that Theorem 1 applied to $G=K_{n}$ and $\kappa \equiv 1$ translates to Hall's theorem for a system of $n$ distinct representatives.

Bollobás and Varopoulos [2] generalized Hall's theorem to measurable sets. In particular, they proved the following.

Theorem 2 (Bollobás and Varopoulos [2]). Let $(X, \mathcal{M}, \mu)$ be an atomless measure space, let $\left(X_{\alpha}\right)_{\alpha \in A}$ be a family of measurable sets of $\mathcal{M}$ with (finite or infinite) index set $A$, and let $\kappa: A \rightarrow \mathbb{R}^{+}$. If for every finite subset $B$ of $A$,

$$
\sum_{\alpha \in B} \kappa(\alpha) \leq \mu\left(\bigcup_{\alpha \in B} X_{\alpha}\right),
$$

then there exists a family $\left(Y_{\alpha}\right)_{\alpha \in A}$ of measurable sets satisfying $Y_{\alpha} \subseteq X_{\alpha}$ and $\mu\left(Y_{\alpha}\right)=\kappa(\alpha)$ for every $\alpha \in A$, and $\mu\left(Y_{\alpha} \cap Y_{\alpha^{\prime}}\right)=0$ whenever $\alpha \neq \alpha^{\prime}$.

Inspired by Theorems 1 and 2, Hilton and Johnson [5] considered, for finite graphs, a common generalization of both these theorems. For an atomless measure space $(X, \mathcal{M}, \mu)$ and for demands $\kappa: V(G) \rightarrow \mathbb{R}^{+}$, an assignment $L(v) \in \mathcal{M}$ satisfies the generalized Hall condition if

$$
\sum_{v \in V(H)} \kappa(v) \leq \int_{X} \alpha(x, L, H) \mathrm{d} \mu
$$

holds for every subgraph $H$ of $G$. Note that the function $\alpha(x, L, H)$ is integrable since $L(v) \in \mathcal{M}$ for every vertex $v$. Hilton and Johnson [5] proved the following.

Theorem 3 (Measurable CGL Theorem [5]). The following statements are equivalent for a finite graph $G$. 
- For every $\kappa: V(G) \rightarrow \mathbb{R}^{+}$, an atomless measure space $(X, \mathcal{M}, \mu)$ and $L: V(G) \rightarrow \mathcal{M}$, the generalized Hall condition (2) is sufficient and necessary for the existence of a coloring $c: V(G) \rightarrow \mathcal{M}$ such that for every $v \in V(G), c(v)$ is a subset of $L(v)$ of measure $\kappa(v)$, and $c(v) \cap c\left(v^{\prime}\right)=\emptyset$ for every pair of adjacent vertices $v$ and $v^{\prime}$;

- $G$ is the line graph of a forest;

- every block of $G$ is a clique and each cut-vertex belongs exactly to two blocks;

- $G$ does not contain $K_{4}^{-}, K_{1,3}$ or a cycle of length more than 3 as an induced subgraph.

The proof of the harder implication of Theorem 3 given by Hilton and Johnson [5] is six pages long, and it is quite involved; we present a shorter proof in Section 3.

\section{Notation}

We introduce notation used throughout the paper. All measure spaces considered are atomless. Carathéodory established that if $(X, \mathcal{M}, \mu)$ is such a measure space and $A \in \mathcal{M}$, then for every real $\beta \in(0, \mu(A))$ there exists a measurable set $B \subset A$ such that $\mu(B)=\beta$. The reader is referred to the book of Fremlin [4] for more details.

It turns out that in our considerations it is useful to work with demand and list size functions both in the measure and the ordinary setting. Let us first introduce the notation for multicolorings. Let $\kappa: V(G) \rightarrow \mathbb{R}^{+}$and $\lambda: V(G) \rightarrow \mathbb{R}^{+}$be two functions from the vertex set of a graph $G$ to nonnegative real numbers. A $\lambda$-list assignment is a function $L$ that assigns to each vertex $v$ of $G$ a set $L(v)$ of at least $\lambda(v)$ colors. A mapping $c$ that assigns to each vertex a set $c(v) \subseteq L(v)$ is a $\kappa$-coloring with respect to $L$ if $|c(v)| \geq \kappa(v)$ for every vertex $v \in V(G)$, and $c(v) \cap c\left(v^{\prime}\right)=\emptyset$ for every pair of adjacent vertices $v$ and $v^{\prime}$. If $G$ admits a $\kappa$-coloring with respect to every $\lambda$-list assignment, we say that $G$ is $(\kappa, \lambda)$-choosable.

The just introduced definitions readily translate to measurable colorings. A measurable $\lambda$-list assignment is a mapping $L: V(G) \rightarrow \mathcal{M}$ such that $\mu(L(v)) \geq \lambda(v)$ for every vertex $v$. A mapping $c$ that assigns to each vertex 
a measurable set $c(v) \subseteq L(v)$ is a measurable $\kappa$-coloring with respect to $L$ if $\mu(c(v)) \geq \kappa(v)$ for every $v \in V(G)$ and $c(v) \cap c\left(v^{\prime}\right)=\emptyset$ for every pair of adjacent vertices $v$ and $v^{\prime}$. The graph $G$ is measurably $(\kappa, \lambda)$-choosable if $G$ has a measurable $\kappa$-coloring for every measurable $\lambda$-list assignment. We also slightly extend our notation and for $t \in \mathbb{R}^{+}$, we call $c$ a $t$-coloring if it is a $\kappa$-coloring where $\kappa \equiv t$. Similarly, we define a $t$-list assignment. Observe that using this extended notation, the measurable list chromatic number of $G$ is the smallest $t$ such that $G$ has a measurable 1-coloring for every measurable $t$-list assignment.

\section{Proof of the Measurable CGL Theorem}

In this section, we provide an alternative proof of Theorem 3.

Proof of Theorem 3. If $G$ contains $K_{4}^{-}, K_{1,3}$ or a cycle of length more than three as an induced subgraph, then a measurable list assignment $L$ and a mapping $\kappa: V(G) \rightarrow \mathbb{R}^{+}$can be constructed [5] such that the generalized Hall condition is satisfied and $G$ has no $\kappa$-coloring with respect to $L$.

In the rest, we focus on proving that if $G$ contains no $K_{4}^{-}$, no $K_{1,3}$ and no cycle of length more than three as induced subgraphs, then $G$ is measurably $\kappa$-choosable for a list assignment $L$ whenever $\kappa$ and $L$ satisfy the generalized Hall condition. For the proof, let us fix an atomless measure space $(X, \mathcal{M}, \mu)$, a mapping $\kappa: V(G) \rightarrow \mathbb{R}^{+}$and a list assignment $L: V(G) \rightarrow \mathcal{M}$ that satisfy the generalized Hall condition. Let $\varepsilon_{i}=\frac{1}{i+1}$ for each integer $i$.

Let $\bar{L}: 2^{V(G)} \rightarrow \mathcal{M}$ be the mapping that assigns to each subset $U$ of $V(G)$ the set of those elements of $X$ contained in all the sets $L(u)$ for $u \in U$, and in none of the sets $L(u)$ for $u \notin U$. Formally,

$$
\bar{L}(U)=\left(\bigcap_{u \in U} L(u)\right) \backslash\left(\bigcup_{u \notin U} L(u)\right) .
$$

Let

$$
x_{i}=\frac{\varepsilon_{i}}{2} \cdot \min \left\{\min _{\substack{v \in V(G) \\ \kappa(v)>0}} \kappa(v), \min _{\substack{U \subseteq V(G) \\ \mu(\bar{L}(U))>0}} \mu(\bar{L}(U))\right\} .
$$

The value of $x_{i}$ represents the measure corresponding to one color in the list assignment defined in the sequel. For each $U \subseteq V(G)$, fix a set $A_{i, U}$ of 
$\left\lfloor\mu(\bar{L}(U)) / x_{i}\right\rfloor$ colors so that the sets $A_{i, U}$ are disjoint for different choices of $U$. By the definition of $x_{i}$, it holds that

$$
\left|A_{i, U}\right| \geq \frac{\mu(\bar{L}(U))}{x_{i}}-1 \geq\left(1-\frac{\varepsilon_{i}}{2}\right) \frac{\mu(\bar{L}(U))}{x_{i}} .
$$

We define the list assignment $L_{i}^{\prime}$ by setting

$$
L_{i}^{\prime}(v)=\bigcup_{\substack{U \subseteq V(G) \\ v \in U}} A_{i, U}
$$

for every $v \in V(G)$. Note that $\left|L_{i}^{\prime}(v)\right| \geq\left(1-\varepsilon_{i} / 2\right) \mu(L(v)) / x_{i}$ by (4). Finally, define $\kappa_{i}^{\prime}(v)=\left\lfloor\left(1-\varepsilon_{i} / 2\right) \kappa(v) / x_{i}\right\rfloor$ and observe that $\kappa_{i}^{\prime}(v) \geq\left(1-\varepsilon_{i}\right) \kappa(v) / x_{i}$ by the choice of $x_{i}$ in $(3)$.

We verify that the function $\kappa_{i}^{\prime}$ and the list assignment $L_{i}^{\prime}$ satisfy the Hall condition. Consider a subgraph $H$ of $G$. Since $L$ and $\kappa$ satisfy the generalized Hall condition, it holds that

$$
\sum_{v \in V(H)} \kappa(v) \leq \int_{x} \alpha(x, L, H) \mathrm{d} \mu(x)=\sum_{U \subseteq V(G)} \alpha(H[U \cap V(H)]) \cdot \mu(\bar{L}(U)) .
$$

We infer from (4) that

$$
\sum_{v \in V(H)} \kappa(v) \leq \frac{x_{i}}{1-\varepsilon_{i} / 2} \sum_{U \subseteq V(G)} \alpha(H[U \cap V(H)]) \cdot\left|A_{i, U}\right|,
$$

and consequently that

$$
\begin{aligned}
\sum_{v \in V(H)} \kappa^{\prime}(v) & =\sum_{v \in V(H)}\left\lfloor\frac{1-\varepsilon_{i} / 2}{x_{i}} \kappa(v)\left|\leq \sum_{U \subseteq V(G)} \alpha(H[U \cap V(H)]) \cdot\right| A_{i, U} \mid\right. \\
& =\sum_{x \in \cup_{v \in V(H)}} \alpha\left(x, L^{\prime}, H\right) .
\end{aligned}
$$

Hence, $\kappa_{i}^{\prime}$ and $L_{i}^{\prime}$ satisfy the Hall condition. By Theorem 1, there exists a $\kappa_{i}^{\prime}$-coloring $c_{i}$ for the list assignment $L_{i}^{\prime}$.

For every $U, W \subseteq V(G)$, we define $\sigma_{i}(U, W)$ to be the number of elements of $A_{i, U}$ assigned by $c_{i}$ precisely to the vertices contained in $W$. In addition, we let $\sigma_{i}^{\prime}(U, W)=\sigma_{i}(U, W) x_{i}$. 
Each of the functions $\sigma_{i}^{\prime}$ can be viewed as a $2^{2|V(G)|}$-dimensional vector of real numbers from the interval $\left[0, \max _{v \in V(G)} \mu(L(v))\right]$. Using this correspondence, the functions $\sigma_{i}^{\prime}$ form a sequence of $2^{2|V(G)|}$-dimensional vectors contained in a bounded and closed subspace of $\mathbb{R}^{2^{2|V(G)|}}$. Thus, by the theorem of Heine-Borel, there exists a converging subsequence of $\sigma_{i}^{\prime}$. Let $\sigma(U, W)$ be the function equal to such a limit vector.

As $\left|c_{i}(u)\right| \geq\left(1-\varepsilon_{i}\right) \kappa(u) / x_{i}$ for every vertex $u$ and every index $i$, we deduce that

$$
\sum_{\substack{U, W \subseteq V(G) \\ u \in W}} \sigma(U, W) \geq \kappa(u),
$$

for every vertex $u$. Furthermore, for each non-empty subset $U$ of $V(G)$

$$
\sum_{W \subseteq V(G)} \sigma(U, W) \leq \mu(\bar{L}(U)),
$$

since $\sum_{W \subseteq V(G)} \sigma_{i}(U, W) \leq \mu(\bar{L}(U))$ for every index $i$. Consequently, as the considered measure space is atomless, there exist disjoint subsets $K_{U, W}$ of $\bar{L}(U)$ such that $\mu\left(K_{U, W}\right)=\sigma(U, W)$. If $\sigma(U, W)=0$, set $K_{U, W}=\emptyset$. Observe that the subsets $K_{U, W}$ are pairwise disjoint, as the sets $\bar{L}(U)$ are.

We are now ready to define the desired measurable coloring. For every vertex $u$ of $G$, we set

$$
c(u)=\bigcup_{\substack{U, W \subseteq V(G) \\ u \in W}} K_{U, W} .
$$

It follows from (7) that $\mu(c(u)) \geq \kappa(u)$ for every vertex $u$, as the sets $K_{U, W}$ are pairwise disjoint. It remains to argue that the obtained coloring is proper and that each vertex is colored with a subset of its list.

Let $u$ and $u^{\prime}$ be two adjacent vertices. Observe that for all subsets $U$ and $W$ of $V(G)$ such that $\left\{u, u^{\prime}\right\} \subseteq W$, it holds that $\sigma_{i}^{\prime}(U, W)=0$ for every index $i$. Hence, $\sigma(U, W)=0$ and thus $K_{U, W}=\emptyset$. Since the sets $K_{U, W}$ are pairwise disjoint, we infer that $c(u) \cap c\left(u^{\prime}\right)=\emptyset$.

It remains to show that $c(u) \subseteq L(u)$ for every vertex $u$. Indeed, since $c(u)$ is a union of sets $K_{U, W}$ such that $u \in W \subseteq U$ and $K_{U, W} \subseteq \bar{L}(U)$, the inclusion $c(u) \subseteq L(u)$ follows by the definition of $\bar{L}(U)$. 


\section{Fractional colorings}

As mentioned in the Introduction, Alon et al. [1] showed that the fractional chromatic number and the list fractional chromatic number are the same. Here, we follow the lines of their proof and show that the fractional chromatic and the measurable list chromatic numbers are the same.

Theorem 4. If a finite graph $G$ has fractional chromatic number $\chi_{f}$, then $G$ is measurably $\left(1, \chi_{f}\right)$-choosable. In particular, $\chi_{m, \ell}(G)=\chi_{f}$.

Proof. Fix a graph $G$. Since $G$ is finite, there exist two integers $p$ and $q$ such that $\chi_{f}=p / q$. Thus, there exists a $q$-coloring $c_{f}: V(G) \rightarrow\left(\begin{array}{c}\{1, \ldots, p\} \\ q\end{array}\right)$. Next, fix an atomless measure space $(X, \mathcal{M}, \mu)$ with $\mu(X) \geq \mu$, and a measurable $\chi_{f}$-list assignment $L: V(G) \rightarrow \mathcal{M}$.

We now define a partition of $X$ into $p$ (measurable) subsets $X_{1}, \ldots, X_{p}$. For every subset $U \subseteq V(G)$, let $Y^{U} \subseteq X$ be the set of those colors contained precisely in all the lists $L(v), v \in U$, and none of the lists $L(v), v \notin U$. Note that the sets $Y^{U}$ are disjoint for different choices of $U \subseteq V(G)$. Since the considered measure space is atomless, $Y^{U}$ can be partitioned into $p$ measurable sets $Y_{1}^{U}, \ldots, Y_{p}^{U}$ such that $\mu\left(Y_{i}^{U}\right)=\mu\left(Y^{U}\right) / p$. For each $i \in\{1, \ldots, p\}$, the set $X_{i}$ is the union of $Y_{i}^{U}$ taken over all $U \subseteq V(G)$.

By the construction of the partition, $\mu\left(L(v) \cap X_{i}\right)=\mu(L(v)) / p \geq 1 / q$ for each vertex $v$. Indeed,

$$
\mu\left(L(v) \cap X_{i}\right)=\mu\left(\bigcup_{\substack{U \subseteq V(G) \\ v \in U}} Y_{i}^{U}\right)=\sum_{\substack{U \subseteq V(G) \\ v \in U}} \mu\left(Y_{i}^{U}\right)=\sum_{\substack{U \subseteq V(G) \\ v \in U}} \mu\left(Y_{i}\right) / p=\mu(L(v)) / p
$$

We now construct the desired measurable coloring $c: V(G) \rightarrow \mathcal{M}$. Let

$$
c(v)=\bigcup_{k \in c_{f}(v)} L(v) \cap X_{k} .
$$

Since the sets $X_{1}, \ldots, X_{p}$ are mutually disjoint and $c_{f}$ is a fractional coloring of $G, c(v) \cap c\left(v^{\prime}\right)=\emptyset$ for every pair of adjacent vertices $v$ and $v^{\prime}$. Moreover, since $\left|c_{f}(v)\right| \geq q$, the following holds:

$$
\mu(c(v))=\mu\left(\bigcup_{k \in c_{f}(v)} L(v) \cap X_{k}\right)=\sum_{k \in c_{f}(v)} \mu\left(L(v) \cap X_{k}\right) \geq \sum_{k \in c_{f}(v)} 1 / q \geq 1 .
$$

This completes the proof. 


\section{$5 \quad$ Infinite graphs}

In this section, we briefly discuss a possible extension of Theorem 4 to infinite graphs. First, we show that there exists a locally finite bipartite graph $G$ that is not measurably 2-choosable. Note that for every bipartite graph (finite or infinite), both the fractional chromatic number and the measurable chromatic number are equal to two.

Proposition 5. There exists a locally finite bipartite graph $G$ with a countable number of vertices that is not measurably 2-choosable.

Proof. Let us define the graph $G$ as follows.

$$
\begin{aligned}
& V(G)=\bigcup_{i \in \mathbb{N}}\left\{w_{i}^{j}: j=1, \ldots,\left(\begin{array}{c}
2^{i} \\
2^{i-1}
\end{array}\right)\right\} \cup\left\{a_{i}: i \in \mathbb{N}\right\}, \\
& E(G)=\left\{w_{i}^{j} w_{i^{\prime}}^{j^{\prime}}:\left|i-i^{\prime}\right|=1\right\} \cup\left\{a_{i} w_{i}^{j}, a_{i} a_{i+1}: i, j \in \mathbb{N}\right\} .
\end{aligned}
$$

The graph $G$ is a locally finite bipartite graph with a countable number of vertices. The measure space which we consider is the interval $[0,3] \subseteq \mathbb{R}$ enhanced with the Lebesgue measure $\lambda$.

Set $L\left(a_{i}\right)=[0,2]$ for all $i \in \mathbb{N}$. For $i \in \mathbb{N}$, the vertices $\left\{w_{i}^{j} \mid j=\right.$ $\left.1, \ldots,\left(\begin{array}{c}2^{i} \\ 2^{i-1}\end{array}\right)\right\}$ are assigned all sets of the form $S \cup[2,3]$ where $S$ is a subset of the interval $[0,2]$ of measure one that can be written as a union of a finite number of closed intervals of the type $\left[x 2^{-i+1}, y 2^{-i+1}\right]$ where $x, y \in \mathbb{N}_{0}$ (there are $\left(\begin{array}{c}2^{i} \\ 2^{i-1}\end{array}\right)$ choices for $\left.S\right)$.

Suppose now that there is a measurable 1-coloring $c$ of $G$ with $c(v) \subseteq L(v)$ for every vertex $v$ of $G$. By altering the sets $c\left(a_{i}\right)$ by sets of measure zero if needed, we may assume that $c\left(a_{2 i-1}\right)=c\left(a_{2 j-1}\right)$ and $c\left(a_{2 i}\right)=c\left(a_{2 j}\right)$ for all positive integers $i$ and $j$. Furthermore, there exist closed sets $S_{1}$ and $S_{2}$ of measure one that are unions of closed intervals of the type $\left[x 2^{-\ell+1}, y 2^{-\ell+1}\right]$ for some number $\ell \in \mathbb{N}$, and $\lambda\left(S_{1} \cap c\left(a_{1}\right)\right) \geq 3 / 4$ and $\lambda\left(S_{2} \cap c\left(a_{2}\right)\right) \geq 3 / 4$.

We can assume without loss of generality that $\ell$ is odd and consider vertices $w_{\ell}^{i}$ and $w_{\ell+1}^{j}$ such that $S_{1} \subseteq L\left(w_{\ell}^{i}\right)$ and $S_{2} \subseteq L\left(w_{\ell+1}^{j}\right)$. Since $\lambda\left(c\left(a_{\ell}\right) \cap\right.$ $\left.S_{1}\right) \geq 3 / 4$, it follows that $\lambda\left(c\left(w_{\ell}^{i}\right) \cap[2,3]\right) \geq 3 / 4$. Similarly, $\lambda\left(c\left(w_{\ell+1}^{j}\right) \cap\right.$ $[2,3]) \geq 3 / 4$. We conclude that the sets $c\left(w_{\ell}^{i}\right)$ and $c\left(w_{\ell+1}^{j}\right)$ are not disjoint, a contradiction.

We have seen that if $G$ is an infinite graph, then $G$ need not be measurably $\chi_{f}(G)$-choosable. Leader [7] presents the union of Knesser graphs 
$K(3 n, n)$ as an example of a locally finite graph $G$ with $\chi_{f}(G)=\infty$. Note that $\chi_{m}(G)=\chi_{m, l}(G)=3$ in his example. Hence, $\chi_{f}(G) \neq \chi_{m, l}(G)$ even for locally finite graphs, but we believe that if $G$ is locally finite, then $G$ is measurably $\left(\chi_{m}(G)+\varepsilon\right)$-choosable for every $\varepsilon>0$, and thus $\chi_{m}(G)=\chi_{m, l}(G)$.

Conjecture 1. If $G$ is a locally finite graph, then $\chi_{m}(G)=\chi_{m, l}(G)$.

The assumption that $G$ is locally finite is necessary-observe that $K_{\omega, \omega}$, the complete bipartite graph with two countable parts, is not measurably $k$-choosable for any $k \geq 2$ : for a fixed $k$, the lists of the vertices of each part of $K_{\omega, \omega}$ are all finite unions of subintervals of $[0,3 k]$ with rational end points and with measure equal to $k$. The argument that there is no proper measurable coloring follows the lines of the proof of Proposition 5.

\section{Acknowledgment}

The authors are indebted to the referees for their careful reading of the manuscript and suggesting changes that significantly improved the paper. Part of this research was conducted while the first two authors were visiting Georgia Institute of Technology and the middle two authors were visiting Technische Universität Ilmenau. The authors would like to thank their hosts for providing excellent environment for this research, and sharing their mathematical insights.

\section{References}

[1] N. Alon, Zs. Tuza, M. Voigt: Choosability and fractional chromatic numbers, Discrete Math. 165/166 (1997), 31-38.

[2] B. Bollobás, N. Th. Varopoulos: Representation of systems of measurable sets, Math. Proc. Camb. Phil. Soc. 78 (1974), 323-325.

[3] M. Cropper, A. Gyárfás, J. Lehel: Edge list multicoloring trees: an extension of Hall's theorem, J. Graph Theory 42 (2003), 246-255.

[4] D. H. Fremlin: Measure theory. Torres Fremlin, 2002.

[5] A. J. W. Hilton, P. D. Johnson, Jr.: List multicolorings of graphs with measurable sets, J. Graph Theory 54 (2007), 179-193. 
[6] D. G. Hoffman, P. D. Johnson: Extending Hall's theorem into list colorings: a partial history, Int. J. Math. Math. Sci. (2007), Art. ID 72168, 17 pages.

[7] I. Leader: The fractional chromatic number of infinite graphs, J. Graph Theory 20 (1995), 411-417.

[8] E. R. Scheinerman, D. H. Ullman: Fractional graph theory: A rational approach to the theory of graphs. John Wiley \& Sons, 1997, New York. 